\title{
Intravascular Mechanical Thrombectomy for a Patient with Systemic Lupus Erythematosus and Acute Cerebral Infarction with Antiphospholipid Syndrome
}

\author{
Jiaxia $\mathrm{Li}^{1}$, Shugang $\mathrm{Cao}^{1}$, Wei $\mathrm{Hu}^{2}$, Yuyou $\mathrm{Zhu}^{2}$, Mingwu Xia ${ }^{1}$, \\ ${ }^{1}$ Department of Neurology, Hefei Second People's Hospital, Hefei, China \\ ${ }^{2}$ Department of Neurology, The First Affiliated Hospital, University of Science and Technology of China, Hefei, China
}

Email address:

xiamingwu1965@163.com (Mingwu Xia)

${ }^{*}$ Corresponding author

To cite this article:

Jiaxia Li, Shugang Cao, Wei Hu, Yuyou Zhu, Mingwu Xia. Intravascular Mechanical Thrombectomy for a Patient with Systemic Lupus Erythematosus and Acute Cerebral Infarction with Antiphospholipid Syndrome. International Journal of Psychological and Brain Sciences. Vol. 5, No. 1, 2020, pp. 21-27. doi: 10.11648/j.ijpbs.20200501.13

Received: January 19, 2020; Accepted: February 13, 2020; Published: February 20, 2020

\begin{abstract}
Systemic lupus erythematosus (SLE) is a diffuse connective tissue disease that is characterized by autoimmune-mediated inflammation. Antiphospholipid syndrome (APS) is a non-inflammatory autoimmune disease. A 55-year-old female patient was admitted to the hospital due to 'sudden speech insufficiency and aggravation of $5 \mathrm{~h}$ '. The patient developed sudden dizziness, facial numbness, and slurred speech and was transported to the emergency department in a local hospital, and was diagnosed 'cerebral infarction' after a CT examination. According to the medical records, the patient developed SLE at the age of 25. CT showed low density lesions of the left pons and occipital lobe; emergent MRI showed bilateral infarction in the cerebellum and brainstem, and magnetic resonance angiography (MRA) showed upper basilar artery occlusion. Ischemic stroke was diagnosed and NIHSS scored 30. The occluded artery was successfully recanalized with endovascular mechanical thrombectomy. Modified thrombolysis in cerebral infarction (mTICI) blood flow perfusion grade was grade 3 . The case is the first one in the literature that acute ischemic stroke related to SLE vasculitis and antiphospholipid syndrome was treated successfully with endovascular mechanical thrombectomy.
\end{abstract}

Keywords: Thrombolysis, Stroke, Systemic Lupus Erythematosus, Vasculitis

\section{Introduction}

Systemic lupus erythematosus (SLE) is a diffuse connective tissue disease characterized by autoimmune-mediated inflammation. It is mainly characterized by the emergence of antinuclear antibodies (ANA) in serum. A variety of autoantibodies represented by ANA and multiple systems are involved. Antiphospholipid syndrome (APS) is a non-inflammatory autoimmune disease. It is clinically diagnosed with arterial and venous thrombosis and/or pathological pregnancy (early miscarriage and stillbirth) and/or thrombocytopenia as the main manifestation, and the antiphospholipid antibody (aPL) in patient's serum shows positive [1]. The prevalence of ischemic stroke in SLE patients is significantly higher than that in the general population. Its pathogenesis is related to the exfoliation of verrucous endocarditis, vasculitis, and thrombosis caused by atherosclerosis [2-3]. Stroke accounts for $10 \%$ to $15 \%$ of death in SLE patients [4]. APS is prone to cause arteriovenous thrombosis, and its pathogenesis may include: aPL acting on endothelial cells and inducing expression of adhesion molecules and tissue factors; interfering with normal endogenous anticoagulation mechanisms (inhibition of protein $\mathrm{C}$ pathway; inhibition of antithrombin); activating complement cascade reaction; binding and activating platelets [5]. Treatment of acute ischemic stroke in patients with SLE and APS is limited. There are reports that by using alteplase in the treatment of acute middle cerebral artery occlusion with SLE, the blood vessels are effectively recanalized and the outcome is good [6-8]. However, the main disadvantages of intravenous thrombolysis are that the time window is narrow, the contraindications are relatively large with high risk of 
bleeding, and the recanalization rate of acute ischemic stroke caused by large vessel occlusion is not high [9]. A case of SLE combined with APS who was undergoing intravascular mechanical thrombectomy due to concurrent acute basilar occlusion with thrombocytopenia is reported. It is hoped that it would provide reference for the treatment of such patients.

\section{Case Report}

\subsection{Clinical Information}

A 55-year-old female patient was admitted to the First Affiliated Hospital, University of Science and Technology of China due to 'sudden speech insufficiency and aggravation of 5 h'. At 8:10 am on January 29, 2018, the patient developed sudden dizziness, facial numbness, and slurred speech. Then she could not speak, and her right limb was weak. She was admitted to an E.R. in a local hospital, and was diagnosed 'cerebral infarction' after a CT examination. She subsequently had difficulty in breathing and disturbance of consciousness, and was transferred to the First Affiliated Hospital, University of Science and Technology of China at 13:07 after tracheal intubation. Previous medical history: the patient developed SLE at the age of 25, and has been taking prednisone and mycophenolate mofetil. She has a history of hypertension and type II diabetes for 10 years, and claimed no history of ischemic heart disease.

Admission examination: body temperature $37.1^{\circ} \mathrm{C}, 20$ breaths/min, pulse 91 beats/min, blood pressure $134 / 82$ $\mathrm{mmHg}(1 \mathrm{mmHg}=0.133 \mathrm{kPa})$, moderate coma, bilateral pupils were equal round, diameter $2 \mathrm{~mm}$, light reaction was dull, the muscle strength of the right limb was at level zero, Babinski sign on the right was positive, and the left limb was retracted after pain stimulation. The National Institutes of Health Stroke Scale (NIHSS) score was 30 points, and the modified Rankin Scale (mRS) score was 5 points.

Laboratory examination: blood routine showed a white blood cell count of $8.68 \times 109 / \mathrm{L}$, a neutrophil ratio of $90.4 \%$, hemoglobin $70 \mathrm{~g} / \mathrm{L}$, and a platelet count of $57 \times 109 / \mathrm{L}$; C-reactive protein $47.2 \mathrm{mg} / \mathrm{L}$ (normal reference value: 0 to $10.0 \mathrm{mg} / \mathrm{L}$ ); coagulation convention showed prothrombin time was $18.7 \mathrm{~s}$ (normal reference value: 10 to $14 \mathrm{~s}$ ), international normalized ratio was 1.59 , partially activated thromboplastin time was $55.8 \mathrm{~s}$ (normal reference value: 20.0 to $40.0 \mathrm{~s}$ ), fibrinogen concentration was $5.22 \mathrm{~g} / \mathrm{L}$ (normal reference value: 2.00 to $4.00 \mathrm{~g} / \mathrm{L}$ ), thrombin time was $11.0 \mathrm{~s}$ (normal reference value: 14.0 to $21.0 \mathrm{~s}$ ), D-dimer concentration was $1.18 \mathrm{mg} / \mathrm{L}$ (normal reference value: 0 to $0.50 \mathrm{mg} / \mathrm{L}$ ), and red blood cell sedimentation rate was $128 \mathrm{~mm} / \mathrm{h}$ (normal reference value: 0 to $20 \mathrm{~mm} / \mathrm{h}$ ); antinuclear antibody showed positive (quantity $581.009 \mathrm{U} / \mathrm{ml}$ ); blood glucose $15.63 \mathrm{mmol} / \mathrm{L}$; Liver function was normal, serum creatinine was $182 \mu \mathrm{mol} / \mathrm{L}$. Antinucleosome, antihistone and antimitochondrial-M2 were positive, antiß2-glycoprotein I antibody and anticardiolipin antibody were positive. Myeloperoxidase, serine protease 3, anti-glomerular basement membrane antibodies, cytoplasmic type Anti-neutrophil cytoplasmic antibodies and perinuclear anti-neutrophil cytoplasmic antibodies were negative, and serum immunoglobulin, $\operatorname{IgA}, \operatorname{IgM}$, complement $\mathrm{C} 3$, and complement $\mathrm{C} 4$ were normal. There was no abnormality in urinary $\alpha 1$ microglobulin quantification and $24 \mathrm{~h}$ urine protein.

Imaging examination: emergent $\mathrm{CT}$ showed low density lesions of the left pons and occipital lobe; emergent MRI showed bilateral infarction in the cerebellum and brainstem, and magnetic resonance angiography (MRA) showed upper basilar artery occlusion (Figure 1).

\subsection{Evaluation of Surgical Standards}

Indications for intravascular mechanical thrombectomy in patients with basilar artery occlusion are: (1) clinical diagnosis of ischemic stroke and develops measurable neurological deficits such as language, motor function, cognitive impairment, gaze disorder, visual field defects and/or visual neglect within 24 hours of onset; (2) cerebrovascular evaluation indicates the presence of basilar artery occlusion; (3) based on clinical and imaging evaluations, patients are likely to benefit from endovascular treatment; (4) patients have signed the informed consent form. Exclusion criteria: (1) imaging examination revealed intracranial hemorrhage; (2) imaging examination revealed large-scale cerebral infarction (infarction $>2 / 3$ pons or midbrain volume); (3) a clear history of contrast medium allergy; (4) have active bleeding or known bleeding tendency; (5) previous severe disability (mRS score $>3$ points); (6) renal failure, defined as serum creatinine $>177 \mu \mathrm{mol} / \mathrm{L}$ or glomerular filtration rate $<30 \mathrm{ml} /\left(\min \cdot 1.73 \mathrm{~m}^{2}\right)$.

The patient was diagnosed as posterior circulation infarction. Although within the 6-hour effective intravenous thrombolysis time window, the baseline NIHSS score was 30 points, the platelet count was below $100 \times 109 / \mathrm{L}$, the coagulation function was abnormal, and SITS Symptomatic Intracerebral Hemorrhage Risk Score (SICH) was 5 points. Therefore, intravenous thrombolytic therapy was contraindicated [10-11]. With the consent of the patient's family, emergency intravascular mechanical thrombectomy was performed.

\subsection{Surgical Process and Prognosis}

After general anesthesia, the femoral artery was successfully punctured with the Seidinger technique and an $8 \mathrm{~F}$ arterial sheath was placed. The whole brain angiography revealed the occlusion of the upper basilar artery. A $4.0 \mathrm{~mm} \times 20 \mathrm{~mm}$ Solitaire AB stent was inserted along the microcatheter to release it in the P2 segment. The stent was retrieved combined with suction (using a $50 \mathrm{ml}$ syringe connected to the Y-connector when the stent was retrieved, one operator retrieved the stent and the other operator assisted withdrawing the syringe), and one thrombus was removed. The re-examination angiography revealed that a thrombus was visible on the right side of the basilar artery and the posterior cerebral artery was occluded. The stent was pushed into place again using the same method and the stent was retrieved and combined with suction, the second thrombus was removed. Re-examination angiography revealed complete recanalization of the basilar artery and the left posterior cerebral artery (Figure 2). Vascular recanalization time was 16:19, and modified 
thrombolysis in cerebral infarction (mTICI) blood flow perfusion grade was grade 3 . XperCT examination showed no significant sign of intracranial hemorrhage after operation (Figure 3). Pathological examination of the thrombi obtained after surgery revealed red thrombi, main components of which were red blood cells, white blood cells, fibrin, and a small amount of platelets.

The patient was re-examined 90 days later and her speech was unclear, with occasionally drinking water choking, limb muscle strength at 5-minus level and was able to take care of herself. Table 1 summarizes the clinical data of this patient.
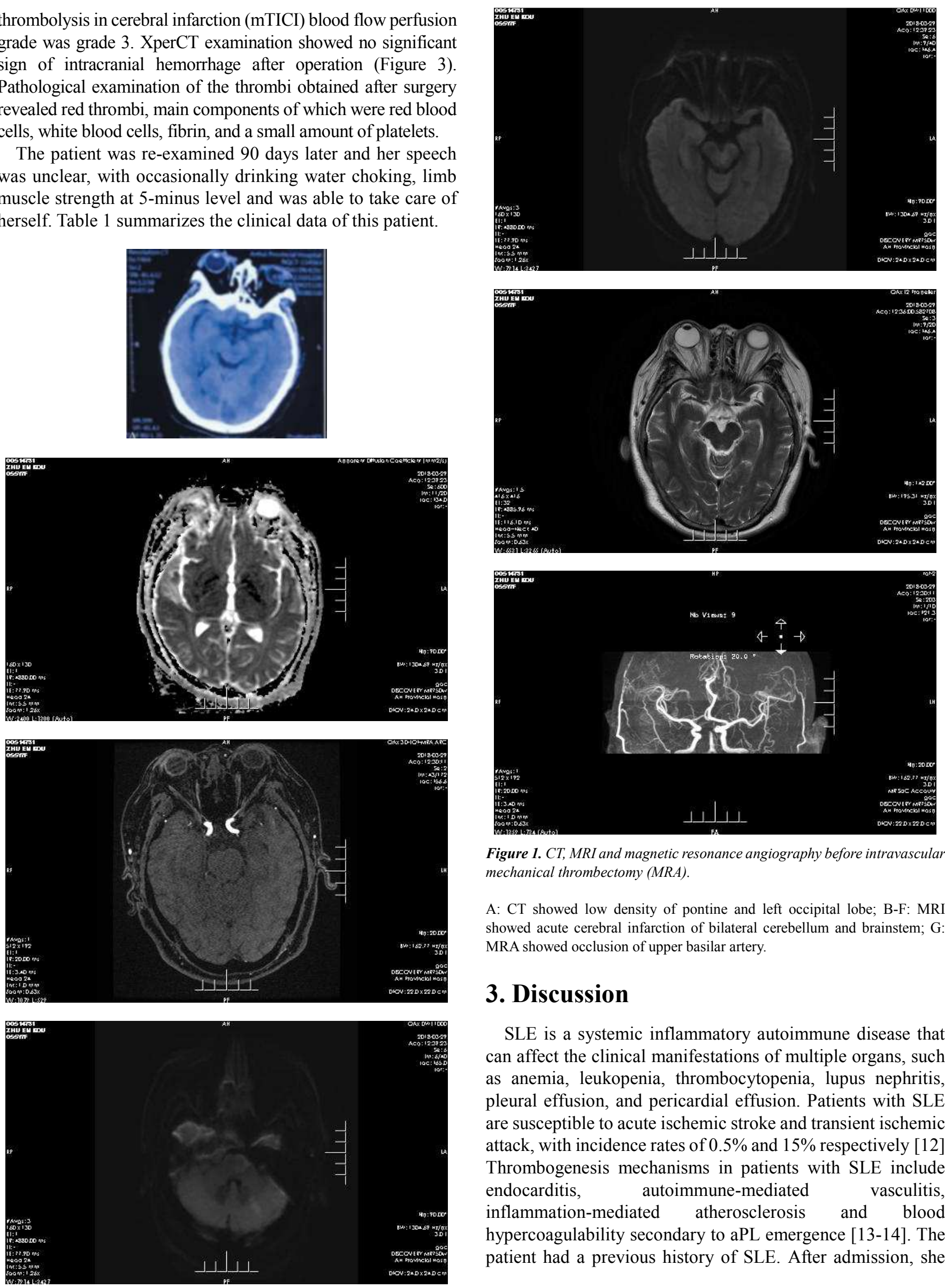

Figure 1. CT, MRI and magnetic resonance angiography before intravascular mechanical thrombectomy (MRA).

A: CT showed low density of pontine and left occipital lobe; B-F: MRI showed acute cerebral infarction of bilateral cerebellum and brainstem; G: MRA showed occlusion of upper basilar artery.

\section{Discussion}

SLE is a systemic inflammatory autoimmune disease that can affect the clinical manifestations of multiple organs, such as anemia, leukopenia, thrombocytopenia, lupus nephritis, pleural effusion, and pericardial effusion. Patients with SLE are susceptible to acute ischemic stroke and transient ischemic attack, with incidence rates of $0.5 \%$ and $15 \%$ respectively [12]. Thrombogenesis mechanisms in patients with SLE include endocarditis, autoimmune-mediated vasculitis, inflammation-mediated atherosclerosis and blood hypercoagulability secondary to aPL emergence [13-14]. The patient had a previous history of SLE. After admission, she 
was positive for antinuclear antibodies, antihistones, anti- $\beta 2$-glycoprotein I antibodies concurrently with anemia, thrombocytopenia, and renal insufficiency. The initial manifestation was dizziness, slurred speech, and gradually progressed to coma with quadriplegia. Considering basilar artery syndrome, cranial CT, MRI and MRA was done and basilar artery occlusion was confirmed. The key to treating acute ischemic stroke is the early recanalization of occluded vessels. The present main methods are intravenous thrombolysis, arterial thrombolysis and intravascular mechanical thrombectomy. Existing studies and guidelines focus on anterior circulation large vessel occlusion, but the latest AHA/ASA guidelines also support endovascular mechanical thrombectomy in patients with basal artery occlusion.
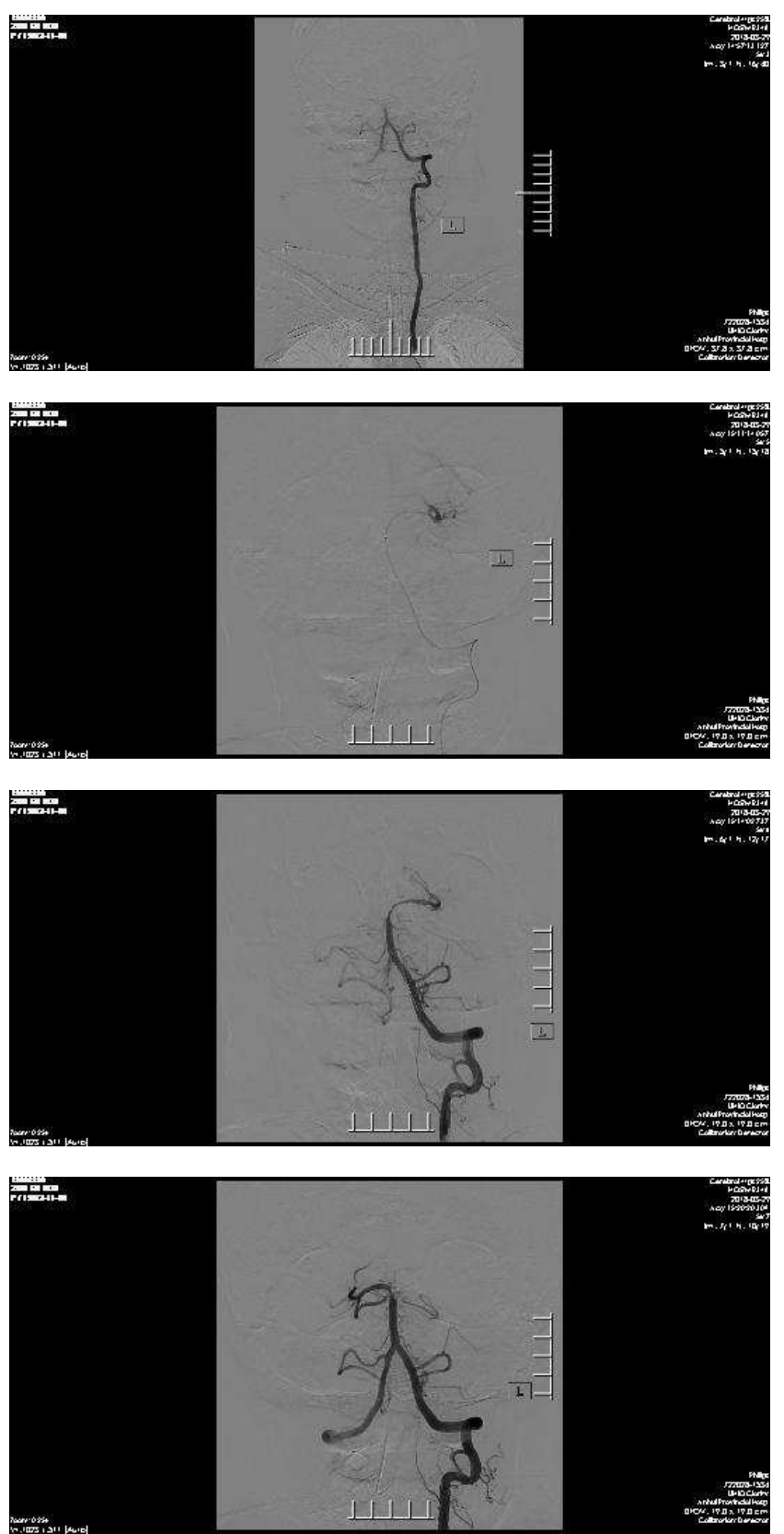

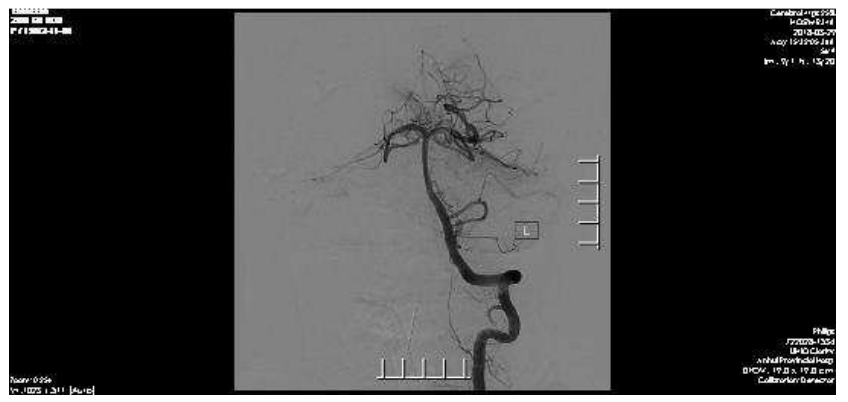

Figure 2. Intravascular mechanical thrombectomy.

A: The left vertebral angiography showed the upper basilar artery occlusion, the distal blood flow was not developed, and the right vertebral blood flow was partially refluxed to the right V3; B: the left posterior cerebral artery P2 segment showed the distal blood Flow was smooth; C: After the first thrombus removal, the angiography showed posterior segment of left posterior cerebral artery P3 and the right posterior cerebral artery was not visualized; D: After the second thrombus removal, posterior segment of right posterior cerebral artery P3 and the left posterior cerebral artery was not visualized; E: A review angiography of the microcatheter suction revealed a complete recanalization of the basilar artery and the left posterior cerebral artery, and occlusion of the $\mathrm{P} 4$ segment of the right posterior cerebral artery. There was no obvious contrast agent exudation.

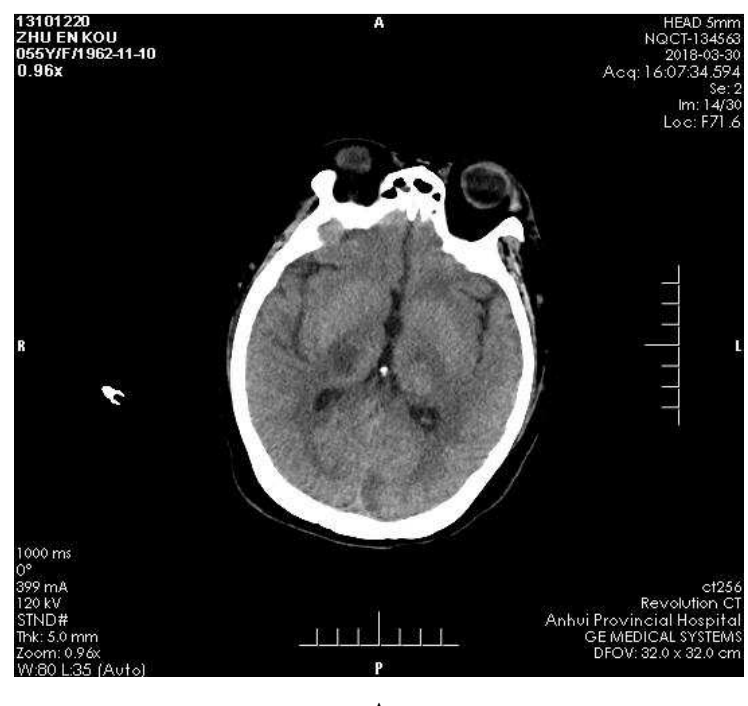

A

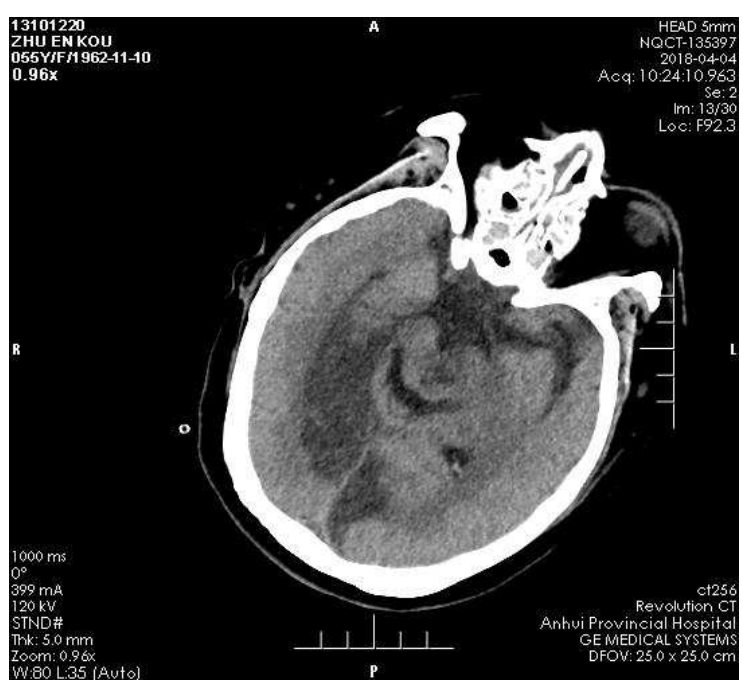




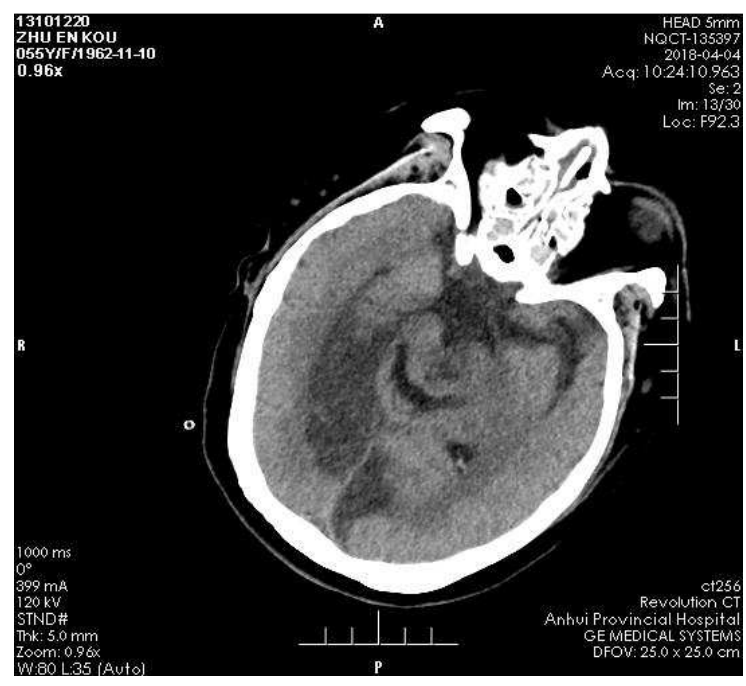

$\mathrm{C}$

Figure 3. CT after Intravascular mechanical thrombectomy.

A: No intracranial hemorrhage lesions were found on the CT scan of the skull after the follow-up examination; B/C: Multiple low-density lesions of the bilateral occipital lobe, bilateral thalamus, bilateral cerebellum, and brain stem were re-examined on the CT scan at 24 hours after the operation.

The basilar artery is the main artery of the vertebrobasilar artery system, with a total length of about $3 \mathrm{~cm}$. It is formed by the convergence of the left and right vertebral arteries at the lower edge of the pons, starting from the midpoint of the pontomedullary sulcus and going up to the basal sulcus of pons. It is divided into left and right posterior cerebral arteries at the midpoint of the pontopontine sulcus. The main branches of the basilar artery are the pontine artery, internal auditory artery, inferior cerebellar artery, superior cerebellar artery, and posterior cerebral artery. They are responsible for blood supply to the posterior part of the brain including the brain stem, cerebellum, thalamus, and temporal occipital lobe. Occlusion at early stage may cause symptoms such as dizziness, diplopia, axial ataxia, visual field loss, sensation or dyskinesia, etc. If blood flow cannot be recovered effectively in time, it could cause serious consequences or even death [15-16]. In this case, the patient was diagnosed with vertigo, medulla oblongata, and quadriplegia. Combined with MRI results, the diagnosis of basilar artery syndrome was definite. How to effectively recanalize occluded blood vessels timely, restore hemodynamics marks, and save the ischemic penumbra is the key to the treatment of ischemic stroke [17]. Solitaire stent produced by EV3 company was originally used for the auxiliary treatment of cerebral aneurysm coil embolism. Its release-repeatable and retrievable characteristics make it capable to remove thrombus, and was approved by the US
Food and Drug Administration as a thrombus removal device for the treatment of ischemic stroke caused by large vessel occlusion. The new Solitaire $\mathrm{AB}$ stent has better maneuverability and plasticity, making thrombus removal more efficient and safe, and effectively preventing thrombus fragments from falling to other blood vessel branches to form new embolisms [18]. At present, this stent is the most mature in the treatment of middle cerebral artery occlusion and thrombectomy, and the high vascular recanalization rate, and the safety and maneuverability have been recognized generally [19-20]. In the past 2 years, more and more studies have reported Solitaire AB stent used in endovascular mechanical thrombectomy for patients with basal artery occlusion, which can improve the vascular recanalization rate of patients with acute basilar artery occlusion with a good rate of patients' outcome and reduce the mortality [21].

It has been reported that 4 patients with SLE complicated with middle cerebral artery occlusion were treated by intravenous thrombolysis with ateplase, and the middle cerebral artery was recanalized effectively without hemorrhagic transformation [7-8]. However, the patient in this case had a baseline NIHSS score of 30 points, a platelet count of $<100 \times 109 / \mathrm{L}$, and a coagulation dysfunction, which is a contraindication for intravenous thrombolytic therapy [9-11]. Therefore, it's more suitble to use arterial thrombolysis or intravascular mechanical thrombectomy. After the consent of the patient's family, the Solitaire $\mathrm{AB}$ stent was used for intravascular mechanical thrombectomy. The cerebral angiography of the patient showed that the basal artery tip thrombus was occluded. In this case, the thrombus could easily oscillate between the left and right posterior cerebral arteries, the blood vessels were difficult to open, and the thrombus could escape and block the distal blood vessels during the process of removing thrombus. Two blood clots were successfully removed from the patient and the recanalization reached mTICI level 3. Postoperative cranial CT showed no obvious bleeding lesions, indicating that no intracranial hyperperfusion bleeding occurred and the operation was successful. Pathological examination of the thrombus taken showed red thrombus. Combined with a heart color ultrasound detection $24 \mathrm{~h}$ postoperatively which showed the valve having vegetations, thus the cerebral thrombus could have originated from endocarditis. The 90-day postoperative mRS score of the patient was significantly improved compared with the time of admission, indicating that intravascular mechanical thrombectomy is effective in treating patients with SLE complicated with acute basilar occlusion, and this case can provide a reference for the treatment of such patients in the future.

Table 1. Patient's clinical data.

\begin{tabular}{ll}
\hline Characteristics & Values \\
\hline Gender & Female \\
Age & 55 \\
History of hypertension & 10 years \\
History of type II diabetes & 10 years \\
History of systemic lupus erythematosus & 30 years \\
\hline
\end{tabular}




\begin{tabular}{ll}
\hline Characteristics & Values \\
\hline Baseline NIHSS score & 30 \\
Baseline mRS score & 5 \\
Time from onset of symptoms to admission & $300 \mathrm{~min}$ \\
Time from admission to puncture & $153 \mathrm{~min}$ \\
Time from puncture to recanalization & $39 \mathrm{~min}$ \\
Time from onset of symptoms to recanalization & $492 \mathrm{~min}$ \\
Stroke type & Thromboembolism \\
Treatment programs & Endovascular mechanical thrombectomy \\
Number of thrombectomy & 2 \\
Recanalization grading & mTICI level 3 \\
Symptomatic cerebral hemorrhage & No \\
24h NIHSS score postoperative & 26 \\
72h NIHSS score postoperative & 20 \\
30d NIHSS score postoperative & 16 \\
90d mRS score postoperative & 2 \\
\hline
\end{tabular}

+NIHSS: National Institutes of Health Stroke Scale; mRS: Modified Rankin Scale; mTICI: Modified Blood Flow Perfusion Classification Standard for Cerebral Infarction.

\section{Conclusion}

In this rare case, endovascular mechanical thrombectomy was suitable for patients with Systemic Lupus Erythematosus and Antiphospholipid Syndrome complicated by acute basilar artery occlusion. Our case achieved a good prognosis by emergency mechanical thrombectomy, and can provide a guidance for such patients in the future.

\section{Acknowledgements}

This work was supported by the Key Research and Development Plan Projects of Anhui Province (1804h08020233); Hefei Municipal Health and Family Planning Commission Applied Medical Research Project (hwk2017zd008). We are grateful for the technical support and case provision of the First Affiliated Hospital of University of Science and Technology of China. We thank Director Xia Mingwu and Dr. Cao Shugang for participating.

\section{References}

[1] Chinese Medical Association Rheumatology Branch. Guidelines for the diagnosis and treatment of systemic lupus erythematosus [J]. Chinese Journal of Rheumatology, 2010, 14 (5): $342-346$.

[2] Chen, X., \& Xu, G. (2018). Intravenous thrombolysis in SLE-related stroke: a case report and literature review. Neurological Sciences, 39 (1), 155-159.

[3] Wiseman, S. J., Bastin, M. E., Jardine, C. L., Barclay, G., Hamilton, I. F., Sandeman, E., \& Ralston, S. H. (2016). Cerebral small vessel disease burden is increased in systemic lupus erythematosus. Stroke, 47 (11), 2722-2728.

[4] Holmqvist, M., Simard, J. F., Asplund, K., \& Arkema, E. V. (2015). Stroke in systemic lupus erythematosus: a meta-analysis of population-based cohort studies. RMD open, 1 (1), e000168.

[5] Lim, W. (2009). Antiphospholipid antibody syndrome. Hematology Am Soc Hematol Educ Program, 233-239.
[6] Loharia, J. J., Alam, J. M., Abdelhadi, H. A., \& Marei, T. F. (2015). Thrombolytic therapy at systemic lupus onset with secondary antiphospholipid syndrome: A rare stroke experience. Neurosciences, 20 (1), 55.

[7] Camara-Lemarroy, C. R., Infante-Valenzuela, A., Andrade-Vazquez, C. J., Enriquez-Noyola, R. V., Garcia-Valadez, E. A., \& Gongora-Rivera, F. (2016). Successful intravenous thrombolysis in a patient with antiphospholipid syndrome, acute ischemic stroke and severe thrombocytopenia. Blood Coagulation \& Fibrinolysis, 27 (3), 354-356.

[8] Majdak, M. R., \& Vuletić, V. (2016). Thrombolysis for acute stroke in patient with systemic lupus erythematosus: A case report. Journal of the neurological sciences, 361, 7-8.

[9] Khatri, R., Vellipuram, A. R., Maud, A., Cruz-Flores, S., \& Rodriguez, G. J. (2018). Current endovascular approach to the management of acute ischemic stroke. Current cardiology reports, 20 (6), 46.

[10] Mazya, M. V., Lees, K. R., Collas, D., Rand, V. M., Mikulik, R., Toni, D. \& Ahmed, N. (2015). IV thrombolysis in very severe and severe ischemic stroke: results from the SITS-ISTR Registry. Neurology, 85 (24), 2098-2106.

[11] Yifeng W., Yun X., Xueling Z., Junshan Z., Hongchao S., \& Jingwei L. (2018). Comparison of safety and effectiveness of direct thrombectomy and bridging therapy within 4.5 hours of stroke in acute large vessel occlusion. International Journal of Cerebrovascular Disease, 26 (4): 257 -261.

[12] Fernández-Nebro, A., Rúa-Figueroa, Í., Lopez-Longo, F. J., Galindo-Izquierdo, M., Calvo-Alén, J., Olivé-Marqués, A.,... \& Ibáñez-Rúan, J. (2015). Cardiovascular events in systemic lupus erythematosus: a nationwide study in Spain from the RELESSER registry. Medicine, 94 (29).

[13] Wick, G., Schett, G., Amberger, A., Kleindienst, R., \& Xu, Q. (1995). Is atherosclerosis an immunologically mediated disease?. Immunology today, 16 (1), 27-33.

[14] Kabakov, A. E., Tertov, V. V., Saenko, V. A., Poverenny, A. M., \& Orekhov, A. N. (1992). The atherogenic effect of lupus sera: systemic lupus erythematosus-derived immune complexes stimulate the accumulation of cholesterol in cultured smooth muscle cells from human aorta. Clinical immunology and immunopathology, 63 (3), 214-220. 
[15] Cerrato, P., Berardino, M., Bottacchi, E., Corso, G., Lentini, A., Bosco, G. \& Bergui, M. (2008). Vertebral artery dissection complicated by basilar artery occlusion successfully treated with intra-arterial thrombolysis: three case reports. Neurological Sciences, 29 (1), 51-55.

[16] Mattle, H. P., Arnold, M., Lindsberg, P. J., Schonewille, W. J., \& Schroth, G. (2011). Basilar artery occlusion. The Lancet Neurology, 10 (11), 1002-1014.

[17] Bhatti, A., \& Sivakumaran, P. (2017). Intra-arterial Treatment for Basilar Artery Occlusion. JAMA neurology, 74 (1), 130-130.

[18] Park, H., jun Hwang, G., Jin, S. C., Jung, C. K., Bang, J. S., Han, M. K.,... \& Kwon, O. K. (2011). A retrieval thrombectomy technique with the Solitaire stent in a large cerebral artery occlusion. Acta neurochirurgica, 153 (8), 1625-1631.
[19] Gory, B., Sivan-Hoffmann, R., Riva, R., Labeyrie, P. E., Huguet, N., Nighoghossian, N., \& Turjman, F. (2015). Repeated Solitaire mechanical thrombectomy in an acute anterior stroke patient. Revue neurologique, 171 (8-9), 682.

[20] McCabe, J. J., Phillips, T. J., Phatouros, C., Singh, T., Blacker, D., Hankey, G. J., \& McAuliffe, W. (2013). Mechanical thrombectomy with the Solitaire $\mathrm{AB}$ device in large intracerebral artery occlusions. Journal of medical imaging and radiation oncology, 57 (2), 149-155.

[21] Chunxia Z., Wanchao S., Futang X., \& Chen L. (2018). Solitaire $\mathrm{AB}$ stent mechanical thrombectomy for acute ischemic stroke: comparison of cardiogenic embolism and aortic atherosclerotic stroke. International Journal of Cerebrovascular Disease, 26 (6): 401-406. 